\title{
Al minds need to think about energy constraints
}

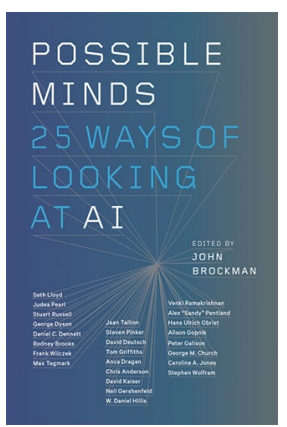

same time emphasize that the societal context of AI mostly remains as Norbert Wiener painted it.

The problem of goal formulation for AI is a motif throughout the book, especially so in the context of value alignment between humans and AI. A suggestive resolution emerges from multiple essays - instead of providing explicit learning objectives for AI, could the way forward be to learn these objectives from examples? This is implied to be similar to reinforcement learning in reverse, or, perhaps, meta-reinforcement learning: an AI could infer the objectives of the rewarder by observing their rewards. Some initial research in this direction is already available ${ }^{2}$.

While the experts of Possible Minds do not realistically expect AI to control the world anytime soon - at least not in any malicious sense - the perception of continued progress is in the air. So much (and at the same time, so little) progress towards intelligence has already been achieved by AI. DeepBlue, AlphaGo and classifying cat images are often emphasized as tasks that are sufficiently mastered, but narrowly specialized. Despite robot scientists making the news ${ }^{3}$, tasks that require creativity, conceptual novelty and curiosity are still out of reach.

Possible Minds delivers a contemporary summary of where machine intelligence stands. There is, however, one perspective that I found absent throughout the book: that of energy. All organisms in the natural world are constantly striving to acquire and control expansive energy for growth and reproduction. Why would machine intelligence be different? Any autonomous, superintelligent AI - either friendly or malicious - would need energy, regardless of the technology used.
Most of the future scenarios in the book imply that intelligent thinking carries no energy cost, or that these costs are negligible. An exception is George Church's essay (chapter 23), where he notes that Watson - winner of Jeopardy! - used 85,000 W of energy in real time, while the human brains it was competing against were using $20 \mathrm{~W}$ each - three orders of magnitude more energy. A recent study found that the carbon footprint of training a single deep learning model may be larger than the entire lifetime of several passenger cars ${ }^{4}$.

Humanity today is heavily reliant on energy reserves, consuming annually about one third of all the net primary energy produced on land and shared among all species. In addition, we consume around double that amount from burning fossil fuels ${ }^{5}$, a non-renewable source. When considering 'possible minds' of the future one of the main questions should be: how on Earth will a superintelligent AI find ways to autonomously access and control enormous amounts of extra energy? This remains unanswered in Possible Minds.

\section{Reviewed by Indré Žliobaitè}

University of Helsinki, Helsinki, Finland

e-mail:indre.zliobaite@helsinki.fi

Published online: 31 July 2019

https://doi.org/10.1038/s42256-019-0083-7

References

1. Wiener, N. The Human Use of Human Beings (Riverside, 1950).

2. Leike, J. et al. Preprint at https://arxiv.org/abs/1811.07871 (2018).

3. King, R. D., Schuler Costa, V., Mellingwood, C. \& Soldatova, L. N. IEEE Technol. Soc. Mag. 37, 40-46 (March, 2018).

4. Strubell, E., Ganesh, A. \& McCallum, A. Preprint at https://arxiv. org/abs/1906.02243 (2019).

5. Barnosky, A. MRS Energy Sustain. 2, E10 (2015). 